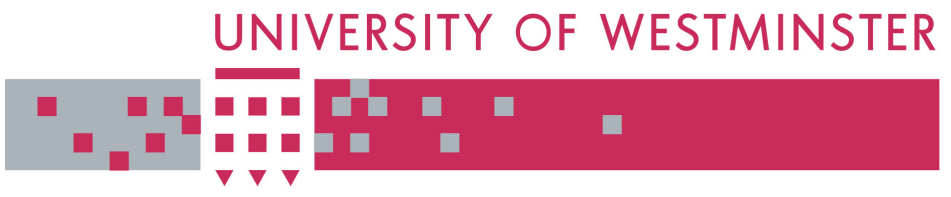

WestminsterResearch

http://www.wmin.ac.uk/westminsterresearch

\title{
Virtual integration of temporal and conflicting information.
}

\section{Panagiotis Chountas}

Department of Computation, UMIST

Ilias Petrounias ${ }^{1}$

Harrow School of Computer Science

${ }^{1}$ At the time of publication llias Petrounias also worked for the Department of Computation, UMIST

Copyright (C [2001] IEEE. Reprinted from 2001 International Database Engineering \& Applications Symposium: proceedings: 16-18 July, 2001, Grenoble, France, pp.243-248.

This material is posted here with permission of the IEEE. Such permission of the IEEE does not in any way imply IEEE endorsement of any of the University of Westminster's products or services. Internal or personal use of this material is permitted. However, permission to reprint/republish this material for advertising or promotional purposes or for creating new collective works for resale or redistribution must be obtained from the IEEE by writing to pubs-permissions@ieee.org. By choosing to view this document, you agree to all provisions of the copyright laws protecting it.

The WestminsterResearch online digital archive at the University of Westminster aims to make the research output of the University available to a wider audience. Copyright and Moral Rights remain with the authors and/or copyright owners. Users are permitted to download and/or print one copy for non-commercial private study or research. Further distribution and any use of material from within this archive for profit-making enterprises or for commercial gain is strictly forbidden.

Whilst further distribution of specific materials from within this archive is forbidden, you may freely distribute the URL of WestminsterResearch. (http://www.wmin.ac.uk/westminsterresearch).

In case of abuse or copyright appearing without permission e-mail wattsn@wmin.ac.uk. 


\section{Virtual Integration of Temporal and Conflicting Information}

\author{
Panagiotis Chountas \\ Department of Computation, \\ UMIST \\ PO Box 88, Manchester M60 1QD, UK \\ E-mail: chountap@sna.co.umist.ac.uk
}

\author{
Ilias Petrounias \\ Department of Computation, \\ UMIST \\ PO Box 88, Manchester M60 1QD, UK \\ E-mail: ilias@sna.co.umist.ac.uk
}

\begin{abstract}
This paper is presenting a way of integrating conflicting temporal information from multiple information providers considering a property-based resolution. The properties considered in this paper are the time and uncertainty because of conflicting information providers. The property based resolution requires a flexible query mechanism, where answers are considered as bounds, taking into account the tendency of things to occur and also the might happen ability of things. Finally some attention is paid to a database environment with non-static members.
\end{abstract}

\section{Introduction}

The integration of information from multiple information providers has been a lasting problem of research. One may distinguish very roughly between two approaches, [1] the lazy and on demand approach. The lazy approach to this problem has been to integrate the independent providers by means of a global conceptual schema that models the information, contained in the entire population of information providers. This global conceptual schema is qualified with a mapping that defines the elements of the global schema, in terms of elements of the schemes of the information providers under conceptual integration. Queries are translated to queries on the population of information providers; the individual answers are then combined to answer the global query. The global schema and the schema mapping constitute the virtual database [2]. A virtual database is that a virtual database points to other databases that contain the data; it does not hold data itself. The basic assumption is that virtual databases should be able to incorporate a large number of information providers, such providers might be used for short time.

Previous work in the area of heterogeneous databases focused on reconciling among different database designs and eventually among different semantics [3], [4]. Information providers may supply contradicting fact instances, which are either time-stamped or not. In the case of time-stamped data, different time dimensions may be considered, such as valid and transaction time. Furthermore if different types of certain temporal information are considered, as defined in [5], [6], conflicts may arise because information providers provide conflicting descriptions in terms of the valid time dimension about the exact duration of a time-stamped fact instance (definite temporal information), the constrained duration of a time-stamped fact instance (indefinite temporal information), the possible known-unknown pair $(K, D)$, where $K$ is the frequency of repetition and $D$ the duration of a periodical or infinite time-stamped fact instance.

The rest of the paper is organised as follows. Section 2 describes the features of a system that supports the integration conflicting information. Section 3 presents the problems in finding the most authoritative answer. Section 4 presents a time model for temporal information. Section 5 defines an extended relational environment where conflicting information is captured. Section 6 presents an extended relational algebra for extraction of flexible answers. Section 7 concludes and investigates the inclusion of contributions coming from new information providers.

\section{Conflict Resolution}

The most important characteristics of a system that supports the integration of conflicting information either temporal or snapshot are described below.

Resolution of Intentional and Extensional Inconsistencies. Intentional inconsistency resolution requires a common format, for all information providers. At this point it is possible that two or more information 
providers could provide conflicting answers to the same query (extensional inconsistency).

Simplicity. There is no restriction on the underlying data model; the only requirement is that results from different providers are concluded in a tabular form.

Minimality. Mappings may neither be total or single valued. In addition to this, ad-hoc queries must be feasible for the capturing of information that is relevant to a given application.

Flexible answers. There is no assumption of mutual consistency between a set of information providers. An authoritative-certain answer may not be possible.

Time property. In terms of the valid time dimension information may be definite, indefinite, and infinite.

Cost Property. The most recently recorded answer is considered to be the most authoritative.

Quality Property. This characteristic may indicate the level of completeness of an answer towards a query.

Uncertainty Property. Conflicting information may generate two types of uncertainty. One is introduced because of queries that refer to level concepts that are at a lower level than those that exist in the instance level of the database. The other arises because of the use of an element in the query that is a member of more than one high level concepts.

\section{Problems in Estimating Flexible Answers}

Let us consider the following description about travellers. "Ann' and 'Liz' "Table 1". Consider the following conflicting queries and whether these could be answered with authority or not:

\begin{tabular}{|c|c|c|c|}
\hline $\mathrm{R}$ & Person & Concept & $\mathrm{VT}(\mathrm{R})$ \\
\hline $\mathrm{X}_{1}$ & Ann & Brazil & {$[01 / 03 / 00,29 / 05 / 00]$} \\
\hline $\mathrm{X}_{2}$ & Ann & $\begin{array}{c}\text { Southern } \\
\text { Hemisphere }\end{array}$ & {$[01 / 06 / 00,29 / 08 / 00]$} \\
\hline $\mathrm{X}_{3}$ & Ann & $\begin{array}{c}\text { Northern } \\
\text { Hemisphere }\end{array}$ & $\begin{array}{l}\{[01 / 06 / 00,29 / 08 / 00], \\
[01 / 09 / 00,29 / 11 / 00]\}\end{array}$ \\
\hline $\mathrm{X}_{4}$ & Ann & Brazil & $\begin{array}{c}{[?, ?]} \\
\text { duration } \\
(90 \text { days somewhere } \\
\text { in year } 2000)\end{array}$ \\
\hline $\mathrm{X}_{5}$ & Liz & Brazil & {$[01 / 03 / 00,29 / 05 / 00]$} \\
\hline $\mathrm{X}_{6}$ & Liz & $\begin{array}{c}\text { Northern } \\
\text { Hemisphere }\end{array}$ & $\begin{array}{c}\{[01 / 06 / 00,29 / 08 / 00], \\
[01 / 09 / 00,29 / 11 / 00]\}\end{array}$ \\
\hline $\mathrm{X}_{7}$ & Liz & Brazil & $\begin{array}{c}{[?, ?]} \\
\text { duration }\end{array}$ \\
& & $\vdots$ & $\begin{array}{c}(90 \text { days somewhere } \\
\text { in year 2000) }\end{array}$ \\
\hline
\end{tabular}

" Table 1: Relation $R$ representing the schedules of Ann and Liz ".
$\mathrm{Q}_{1}$ : When does Liz visit Brazil?

$\mathrm{Q}_{2}$ : When does Ann visit Brazil?

$\mathrm{Q}_{3}$ : Which are all the people who visited the Southern Hemisphere?

$\mathrm{Q}_{4}$ : Which are all the people who visited the Northern Hemisphere?

The above queries are not easy to be answered because of the following inconsistencies: If we consider a latticestructured domain, then Brazil has two parents (Northern Hemisphere and Southern Hemisphere, as shown in "Figure 1". Therefore $Q_{3}$ and $Q_{4}$ are not easy to be answered. We cannot estimate with precision the exact date of arrival and stay in Brazil for both travellers ( $\mathrm{Liz}$, Ann). Therefore $Q_{1}$ and $Q_{2}$ are not easy to be answered either. The above inconsistencies are presented in Table I through relation $R$. An analytical observation of Table I gives rise to the following requirements/issues in terms of data representation:

A time model and representation that presents temporal information in terms of the following physical measurements duration $D$ (e.g. tuples $\left\{\mathbf{X}_{1}, \mathbf{X}_{5}, \mathbf{X}_{2}\right\}$ ) and frequency $\mathrm{K}$ of reappearance, if information is periodical, where $D, K$ may not be known, (e.g. tuples $\left\{\mathbf{X}_{4}, \mathbf{X}_{7}\right\}$ ).

In our effort to classify tuples in Table I based on a virtual decision attribute which simply declares the fact that a person has visited Brazil, it can be seen that tuples $\left\{\mathrm{X}_{2}\right.$, $\left.\mathrm{X}_{3}, \mathrm{X}_{6}\right\}$ cannot be classified with an exclüsive Boolean Yes or No.

Therefore, there is-a need for algebraic operations that will support approximate answers, based on approximation spaces. A similar problem would arise if a user requested through an algebraic operation all the countries that Ann or Liz have visited in the Northern and Southern Hemispheres.

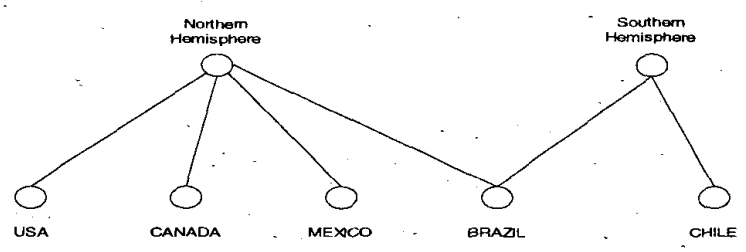

"Figure 1: Lattice Structured Domain "

\section{The Time Model}

In this section the basic elements for a temporal. representation are defined in accordance to [5]. The central concepts are a timeline and a time point where the former is comprised of the latter. The term duration is defined as an absolute distance between two time points. However, the term duration may also imply the existence of two bounds an upper bound and a lower bound (indefinite temporal Information).. $A^{*}$ time interval is 
defined as a temporal constraint over a linear hierarchy of time units denoted $\mathrm{H}_{\mathrm{r} .} \mathrm{H}_{\mathrm{r}}$ is a finite collection of distinct time units, with linear order among those units. For instance, $\mathrm{H}_{1}=$ day $\subseteq$ month $\subseteq$ year, are all linear hierarchies of time units defined over the Gregorian calendar. A time interval is presented in the form of $[C+K \times X, C+K \times X]$ where $C^{\prime}=C+D, D \in N^{*}$, thus an interval is described as a set of two linear equations defined in a linear time hierarchy (e.g. $\mathrm{H}_{2}=$ day $\subseteq$ month $\subseteq$ year).

The lower time point $t_{\text {lower }}$ is described by the equation $\mathrm{t}_{\text {lower }}=C+K^{*} X$. The upper point $\mathrm{t}_{\text {upper }}$ is described by the equation $\mathrm{t}_{\mathrm{upper}}=C^{\prime}+K^{*} X$. $C$ is the time point related to an instantaneous event that triggered a fact, $K$ is the repetition factor, $K \in N^{*}$ or the lexical 'every' (infiniteperiodical information). $X$ is a random variable, $X \in N$, including zero, corresponding to the first occurrence of a fact instance restricted by a constraint. The Sum-Product $K^{*} X+D$ is defined according to a linear hierarchy. $D$ may be in the range between a lower and upper bound $\mathrm{G}_{1} \leq D$ $\leq \mathrm{G}_{2}$ where $\left(\mathrm{G}_{1} \leq \mathrm{G}_{2} \wedge D \leq \mathrm{Y}\right)$. $\mathrm{Y}_{\mathrm{i}} \ldots \mathrm{Y}_{\mathrm{j}}$ are general or restricted constraints on the time points $t_{\text {lower, }} t_{\text {upper- }}$. Constraints are built from arbitrary linear equalities or inequalities (e.g. $\mathrm{t}_{\text {lower }}=C+7 X$ and $0 \leq X \leq 5$ ). Limiting the random variable $X$ results in specifying the lower and upper bound of a time window. The above interval representation permits the expression of the following types of information:

I) Definite Temporal Information: The duration $(D)$ of an event is constant $(D=\mathrm{t})$. All times associated with facts are known precisely in the desired level of granularity. Let $t_{\text {lower, }} t_{\text {upper }}$ be the lower and upper linear points, of a time interval, that determines when a fact instance is defined, in the real world.

$$
\mathrm{t}_{\text {lower }}=C+K^{*} X \quad(1), \mathrm{t}_{\text {upper }}=C^{\prime}+K^{*} X(2), C^{\prime}=C+D \text { (3), }
$$$$
\left(K^{*} X\right)=0(4)
$$

II) Indefinite Temporal Information: is defined when the time associated with a fact has not been fully specified [6]. Therefore the duration of a fact is indeterminate or bounded. This may occur for two reasons: either the duration of a fact is bounded or the duration is known and the start and end point of the time interval are not exactly known. This is defined as following:

$C_{L} \leq C \leq C_{R}(1), D_{L} \leq D \leq D_{R}(2)$

Let $\mathrm{t}_{\text {lower }}, \mathrm{t}_{\text {upper }}$ be the lower and upper linear points, of a time interval, that determines when a fact instance is defined, in the real world.

Adding (2), (1): $C_{L}+D_{L} \leq C \leq C_{R}+D_{R} \Rightarrow C_{L}+D_{L} \leq C^{\prime} \leq$ $C_{R}+D_{R}$ which is the new expression for equation (3) whereas $\left.\left(C_{L}+D_{L}\right) \leq C^{\prime} \leq C_{R}+D_{R}\right) \in H_{r}$. Therefore the time interval (3), that a sample fact instance is defined over, is indeterminate.

III)Infinite Temporal Information: is defined when an infinite number of times are associated with a fact [7].
Infinite temporal information includes the following types of information.

a) Periodic: A fact instance is repeated over a time hierarchy with the following characteristics: a constant frequency of repetition $K$, it has an absolute and constant duration $D$, and $X$ a random variable that denotes the number of reappearances for an event. Therefore the duration of every fact instance constituting a fact type and consequently the duration of a fact type is well known. b)Unknown Recurring Information: Generally is described in the following intervalic form, $t=[\perp, \perp]$. The intuition is that the duration $D$ of an event is assumed to be known or constrained and the frequency of reoccurrence $(K=$ ?) is not known. However by definition it is known that if an event is recurring, then its next reappearance cannot occur before the previous one is ended. Considering Ann's staying to Brazil according to provider $\mathrm{A}_{4}$, she is staying for a period of $(D=90$ days $)$ in Brazil. Using the best case scenario, it can be assumed that Ann is visiting Brazil every $(K=90)$ days, since nothing else is known about Ann's trip to Brazil. It is also known that $K \geq 1$. Therefore the following conclusion can be made $D \leq K$.

It can be also assumed that the time point $C$ related to an instantaneous event that triggered a fact is not known with precision in the time hierarchy $\mathrm{H}_{r}$. $C$ it may be constrained by an application, like as $C_{L} \leq C \leq C_{R}$ or be left unspecified; constraints are part of an answer to a query. In this sense $t_{\text {lower }}, t_{\text {upper }}$ are expressed as following: $\mathrm{t}_{\text {lower }}=K^{*} X+C$ (1) $\quad D \leq K \wedge K \neq 0 K \in N^{*}$ (5)

$\mathrm{t}_{\text {upper }}=K^{*} X+C^{\prime}(2) \quad C_{L} \leq C \leq C_{R}(6)$ $C^{\prime}=C+D(3) \quad D_{L} \leq D \leq D_{R}(7)$

$a_{1} \leq X \leq a_{v}, a_{1}=0$ denotes first occurrence of an event (4) Adding (6), (7) and using (3) it can be deduced that $C_{L}+$ $D_{L} \leq C^{\prime} \leq C_{R}+D_{R}$ (8). (5) is defined through (7) when $D_{L} \leq$ $K \leq D_{R}$ or $K_{L} \leq K \leq K_{R}$ where $D_{L}=K_{L}, D_{R}=K_{R}(9)$. The product $K \times X$ through (9) and (4) is defined as follows

$$
K_{L} *\left(a_{1} \ldots a_{v}\right) \leq K^{*} X \leq K_{R} *\left(a_{1} \ldots a_{v}\right)(10)
$$

Considering $[(8),(6)]$ and $[(8),(10)], t_{\text {lower }}, t_{\text {upper }}$ can be redefined, respectively as follows

$$
\begin{gathered}
\mathrm{t}_{\text {lower }}: K_{L} *\left(a_{1} \ldots a_{v}\right)+C_{L} \leq K^{*} X+C \leq K_{R}^{*}\left(a_{1} \ldots a_{v}\right)+C_{R} \\
\mathrm{t}_{\text {upper }}: K_{L} *\left(a_{1} \ldots a_{v}\right)+C_{L}+D_{L} \leq K^{*} X+C^{\prime} \leq K_{L} *\left(a_{1} \ldots a_{v}\right)+ \\
C_{R}+D_{R}
\end{gathered}
$$

The interpretation is that the time space determined by a recurring event is bounded and consisting of time intervals defined by the lines $\left\{K_{L} *\left(a_{l} \ldots a_{v}\right)+C_{L} \ldots K_{R}{ }^{*}\right.$ $\left.\left(a_{I} \ldots a_{v}\right)+C_{R}\right\}$, for $\mathrm{t}_{\text {lower }}$, the earliest and latest times for a recurring event to start and $\left\{K_{L} *\left(a_{l} \ldots a_{v}\right)+C_{L}+D_{L} \ldots K_{L} *\right.$ $\left.\left(a_{1} \ldots a_{v}\right)+C_{R}+D_{R}\right\}$ for $\mathrm{t}_{\text {upper }}$, the earliest and latest times for a recurring event to be ended, respectively.

However each time line alone, $a_{\lambda}(\lambda \leq v)$, is expressing a separate-monadic periodic event. Proving this argument will enable us to use the above intervalic representation, for expressing periodical facts. In this case the parameters 
$(K, C, D, X)$ are well known. Let us consider for inductive purposes a pair of the above lines named as $t_{L}$ and $t_{R}$, where $\mathrm{t}_{L}=K_{L} *\left(a_{1} \ldots a_{v}\right)+C_{L} \mathrm{t}_{R}=K_{L} *\left(a_{l} \ldots a_{v}\right)+C_{L}+D_{L}$. Proof

For $v=1, t_{L}=K_{L} *\left(a_{1} \ldots a_{l}\right)+C_{L}=C_{L}, t_{R}=K_{L} *\left(a_{1} \ldots a_{1}\right)+$ $C_{L}+D_{L}=C_{L}+D_{L}, a_{1}=0$ denotes the first occurrence of an event. $t=\left[t_{L}, t_{R}\right]$ denotes a time interval that points to the first occurrence of a periodic event.

Let us assume that $t_{L}, t_{R}$ are expressing a periodic event (a) and stand for $\lambda$, occurrences, $\lambda \in N, t_{L(\lambda)}=K_{L}{ }^{*}\left(a_{l} \ldots a_{\lambda}\right)$ $+C_{L}, t_{R(\lambda)}=K_{L} *\left(a_{1} \ldots a_{\lambda}\right)+C_{L}+D_{L}(1)$

If the above argument is correct then, $\mathrm{t}_{\mathrm{L}}, \mathrm{t}_{\mathrm{R}}$ must stand for $\lambda+1$ occurrences, $\lambda \in N$

$\mathrm{t}_{\mathrm{L}(\lambda+1)}=K_{L} *\left(a_{1} \ldots a_{\lambda+1}\right)+C_{L},(2,) \mathrm{t}_{R(\lambda+1)}=K_{L} *\left(a_{1} \ldots a_{\lambda+1}\right)+$ $C_{L}+D_{L}(3), a_{(\lambda+1)}=a_{\lambda}+1(4)$

(2) is rewritten through (1) and (4) as following:

$$
\begin{gathered}
t_{L(\lambda+1)}=K_{L} *\left(a_{1} \ldots a_{\lambda+1}\right)+C_{L}=K_{L} *\left(a_{1} \ldots a_{\lambda}+1\right)+C_{L}= \\
t_{L(\lambda)}+K_{L}
\end{gathered}
$$

(3) is rewritten through (1) and (4) as following:

$$
\begin{gathered}
t_{R(\lambda+1)}=K_{L} *\left(a_{1} \ldots a_{\lambda+1}\right)+C_{L}+D_{L}=K_{L} *\left(a_{1} \ldots a_{\lambda}+1\right)+C_{L} \\
+D_{L}=\mathrm{t}_{\mathrm{R}(\lambda)}+K_{L}
\end{gathered}
$$

The new expressions for (2) and (3) are proving that the $\lambda+l$ occurrences of a periodic event $(a)$ arising from the $\lambda$ ones by adding the characteristic frequency of repetition, which in fact is the definition of a periodic event. "Table 2 " shows the restructuring of Table I according to the proposed model. Next, extended relational algebraic operators are defined for extraction of data, considering matching criteria, that may not be attribute names, or attribute values that may belong to more than one higher conceptual attributes.

\section{Encoding of Conflicting and Uncertain Information}

Definition: Let $T$ be a set of time intervals $T=\left\{\left[t_{L}, t_{R}\right]\right.$ where $\left.t_{L}=C+K^{*} X, t_{R}=C+K^{*} X \wedge a_{1} \leq X \leq a_{v}\right\}$ and $D$ a set of non temporal values. A generalised tuple of temporal arity $x$ and data arity $l$ is an element of $T^{x} \times D^{l}$ together with constraints on the temporal elements. In that sense a tuple can be viewed as defining a potentially infinite set of tuples. Each extended relation consists of generalised tuples as defined above. Each extended relation has a virtual tuple membership attribute formed by a selection predicate either value or temporal that models the necessary $(B e l)$ and possible degrees $(P l s)$ to which a tuple belongs to the relation.

The domain of tuple membership attribute is the Boolean set $\Omega=$ \{true, false $\}$. The possible subsets to that are \{true\}, \{false\} and $\Omega$. The support set for tuple membership can be denoted by a pair of numbers (Bel, $P l s)$ where:

$$
\begin{gathered}
B e l=\mathrm{m}\{\mid \text { truel }\} \\
P l s=\mathrm{m}\{\mid \text { true }\}+\mathrm{m}\{\Omega\} \text { with property } 0 \leq B e l \leq P l s \leq 1
\end{gathered}
$$

A tuple with $(\mathrm{Bel}, \mathrm{Pls})=[1,1]$ corresponds to a tuple that qualifies with full certainty. A tuple with $(\mathrm{Bel}, \mathrm{Pls})=[0,0]$ corresponds to a tuple that is believed not to qualify with full certainty. A tuple with $(\mathrm{Bel}, \mathrm{Pls})=(0,1)$ corresponds to complete ignorance about the tuple's membership. At this point two issues arise: the generalisation of the closed world assumption (CWA) and the estimation of the ( $\mathrm{Bel}$, $P l s$ ) measures.

The CWA is assuming that facts not found in the database are considered to be false. Since tuples memberships in our model vary between $0 \leq$ Bel $\leq P l s \leq 1$ CWA needs to be extended. In generalising the CWA it is assumed that if a fact is not represented in the extended relation, then it must have $B e l=0$, and $P l s \leq 1$. In this sense a database will keep information only if there is some positive evidence about the occurrence of a fact. Therefore, the integrated-virtual database will not contain information of no interest. Using Table $I$ it can be appreciated that using the generalised CWA, the concepts \{Southern Hemisphere, Northern Hemisphere\} will not be replaced by their children as defined in "Figure 1" since there is no support for them. There is only one vote and this can only be capitalised by the high level concepts \{Southern Hemisphere, Northern Hemisphere\}. However, the query "Which are all the people who visited Brazil" is still expecting an answer. In answering this query, all tuples in "Table 2" have to be classified according to the selection predicate location = "Brazil" which forms a virtual attribute (it exists as long as the execution of the query), it is not stored as part of "Table 2". In this sense tuples $\left\{X_{1}, X_{4}, X_{5}, X_{7}\right\}$ are believed to satisfy the virtual attribute location $=$ "Brazil" with certainty $(\mathrm{Bel}, \mathrm{Pls})=$ $[1,1]$. However, it cannot be said with full certainty (Bel, $P l s)=[1,1]$ whether $\left\{\mathrm{X}_{2}, \mathrm{X}_{3}, \mathrm{X}_{6}\right\}$ satisfies the virtual attribute or not. In order to estimate the $(\mathrm{Bel}, \mathrm{Pls})$ measures, the ability to identify higher and lower level concepts for elements defined from a structured domain (either lattice, or tree) as specified by a particular application is needed.

Let $l$ be an element defined by a structured domain $L$. $U(e)$ is the set of higher level concepts, i.e. $U(e)=\{n \mid n \in$ $L \wedge n$ is an ancestor of $l\}$, and $L(e)$ is the set of lower concepts $L(e)=\{n \mid n \in L \wedge n$ is a descendent of $l\}$. If $l$ is a base concept then $L(e)=\varnothing$ and if $l$ is a top level concept, then $U(e)=\varnothing$. If $L$ is an unstructured domain then $L(e)=$ $U(e)=\varnothing$. Considering tuple $\left\{\mathrm{X}_{2}, \mathrm{X}_{3}, \mathrm{X}_{6}\right\}$ and the selection predicate location $=$ "Brazil" then $L(e), U(e)$ are defined as follows:

$U($ Brazil $)=\{$ Southern Hemisphere, Northern Hemisphere $\}, L($ Brazil $)=\varnothing$

Rule 1: If $(|U(e)|>1 \wedge L(e)=\varnothing)$, e.g. $\mid U($ Brazil $) \mid=2$, $L($ Brazil $)=\varnothing$, then it is simply declared that a child or base concept has many parents (lattice structure). Therefore a child or base concept acting as a selection 
predicate can claim any tuple (parent) containing elements found in $U(e)$, as its ancestor, but not with full certainty $(\mathrm{Bel}>0, \mathrm{Pls} \leq \mathrm{I})$. This is presented by the following interval $(\mathrm{Bel}, \mathrm{Pls})=(0,1]$. Now consider the case where the selection predicate is defined as follows location = "Southern Hemisphere".

Tuple $\left\{\mathrm{X}_{2}\right\}$ fully satisfies the selection predicate and thus $(B e l, P l s)=[1,1]$. Tuples $\left\{\mathrm{X}_{3}, \mathrm{X}_{6}\right\}$ do not qualify as an answer and thus $(B e l, P l s)=[0,0]$. However it cannot be said with full certainty $((\mathrm{Bel}, \mathrm{Pls})=[1,1])$ whether $\left\{\mathrm{X}_{1}\right.$, $\left.\mathrm{X}_{4}, \mathrm{X}_{5}, \mathrm{X}_{7}\right\}$ satisfy the selection predicate or not, since Brazil belongs to both concepts \{Southern Hemisphere, Northern Hemisphere\}. Using the functions $L(e), U(e)$ this can be deduced as follows $U($ Southern Hemisphere $)=\{\varnothing\}$

$L($ Southern Hemisphere $)=\{$ Brazil, Chile $\}$

$B=U(L($ Southern Hemisphere $) \wedge($ R.concept $))=U($ Brazil $)$

$=\{$ Southern Hemisphere, Northern Hemisphere $\}$.

Formally the function can be defined as follows: $B\left(\left(l_{l}\right),(\right.$ $\left.\left.l_{2}\right)\right)=U\left(L\left(l_{l}\right) \wedge\left(l_{2}\right)\right)$ where $l_{l}$ is a high level concept, $l_{2}$ is a base concept are elements defined in a lattice structured domain. If both arguments are high level concepts or low level concepts then $B\left(\left(l_{l}\right),\left(l_{2}\right)\right)=\varnothing$. Function $B\left(\left(l_{l}\right),\left(l_{2}\right)\right)$ is defined only in a lattice structured domain.

Rule 2: If $B\left(\left(l_{l}\right),\left(l_{2}\right)\right)$ is defined and $\left|B\left(\left(l_{l}\right),\left(l_{2}\right)\right)\right|>1$, then it is simply declared that multiple parents, high level concepts, are receiving a base concept as their own child. Therefore a parent or high level concept acting as a selection predicate can claim any tuple (child) containing elements found in $\left(L\left(l_{1}\right) \wedge\left(l_{2}\right)\right)$, as its descendant, but not with full certainty ( $\mathrm{Bel}>0, \mathrm{Pl} \leq \mathrm{l}$ ), presented by the following interval $(\mathrm{Bel}, \mathrm{Pls})=(0,1]$. Similarly a temporal selection predicate can use the above functions $(U(e)$, $\left.L(e), B\left(\left(l_{l}\right),\left(l_{2}\right)\right)\right)$ for imprecise temporal information, representing the time dimension as intervals, by labelling each node in the lattice with a time interval. The use of a lattice-structured domain by an application permits also the representation of temporal information at different levels of granularity. Next an extended relational algebra is defined. The operations differ from the traditional ones in several ways: The selection/join condition of the operations may consist of base concepts or high level concepts. Membership threshold $($ Bel $\geq \Phi, P l s)$ may be specified with a selection/join condition to constrain the number of result tuples. The results of extended relational operators either retain or generate the new tuple membership in the case where more than one selection criteria are specified.

\section{Algebraic Operations}

We are considering, for illustration purposes, the four operations $\sigma$ (selection), $\pi$ (projection), $\bowtie$ (join), $\cup$ (set union).
Selection: Selection is defined as follows: $\sigma_{\mathrm{P}}(\mathrm{R}):=\{\mathrm{t}\}$ $t \in R \wedge P(t)=$ true $\}$ where $P$ denotes a selection condition. There are two types of a selection condition. A data selection condition $\left(P_{d}\right)$ considers the snapshot relation $R$ in Table 1. The temporal selection condition $\left(\mathrm{P}_{t}\right)$ is specified as a function of three arguments $\mathrm{P}_{\mathrm{t}}:=\langle K, D, C\rangle$ which is mapped to the time hierarchy $\mathrm{H}_{\mathrm{r}}$ (section 4 ). The relationships between $(K, D)$, depending on the type of temporal information (definite, indefinite, infinite), have been described in section 4 . It has to be mentioned that temporal constraints are included in the result tuples.

The combined predicate over relation VT(R) in "Table 2" is defined as follows: $P:=P_{d}\left|P_{t}\right| P_{d} \wedge P_{t}$. The selection support function $\mathrm{F}_{s}\left(\mathrm{t}_{A l . . A n}, \mathrm{P}\right)$ returns a $(\mathrm{Bel}, \mathrm{Pls})$ pair indicating the support level of tuple $t$ for the selection condition $\mathrm{P}$, where $A_{1} . A_{n}$ is the set of attributes, excluding the virtual membership attribute. The selection support function $\mathrm{F}_{\mathrm{s}}$ utilises the $\left(U(e), L(e), B\left(\left(l_{1}\right),\left(l_{2}\right)\right)\right)$ functions in conjunction with Rule-1 and Rule-2, as defined in section 5, for estimating the actual support values. Recall that a compound predicate is formed by a conjunction of two or more atomic predicates. In this paper it is assumed that the atomic predicates are mutually independent.

The support for the compound predicate $P:=P_{d}\left|P_{t}\right| P_{d} \wedge P_{t}$ is computed based on the multiplicative rule.

$\mathrm{F}_{\mathrm{s}}(\mathrm{P})=\left(\mathrm{F}_{\mathrm{s}}\left(\mathrm{t}_{A l . . A n}, \mathrm{P}_{\mathrm{v}}\right) \wedge \mathrm{F}_{\mathrm{s}}\left(\mathrm{t}_{\mathrm{A} I . . A n}, \mathrm{P}_{\mathrm{d}}\right)\right)=\left(\right.$ Bel $_{1} \times$ Bel $_{2 \ldots . . .} \times B e l_{n}$ , $\left.P l s_{1} \times P l s_{2} \times P l s_{n}\right)(1)$.

A discussion on combining supports of dependent predicates can be found in [8], [9].

Projection: $\pi_{X}(R):=\{t(X) \mid t \in R\}$, where $R$ is a relation on scheme $S, t$ is a tuple with scheme $X$ and $X$ is a subset of $S(X \subseteq S)$. Projection retains all valid time values like standard projection. Projection is defined on top of a selection. The intuition is that, as an operator it does not modify $F_{s}$, that is the tuple membership.

Join: Let $R, S$ be two extended relations, $\mathrm{P}$ be the join condition and $Q$ the membership threshold condition. The extended join operator is defined as a Cartesian Product, followed by an extended selection: $R \bowtie_{\mathrm{p}}{ }^{\mathrm{Q}} S \equiv \sigma_{\mathrm{P}}{ }^{\mathrm{Q}}(\mathrm{R} \times \mathrm{S})$ where the tuple membership function is deviated by $F_{s}(1)$ as in the case of the extended select operation. The time interval that the tuple membership is defined over is the intersection of the time intervals that the sources $\left(A_{1} \ldots A_{n}\right)$ are defined. $\Delta t\left({ }_{F s}\right)=\Delta t_{1} A_{1} \cap \ldots \cap \Delta t_{n} A_{n}$. (2).

Assuming two intervals with lower bounds $t_{L}=C+K X$ and $\mathrm{t}_{L^{\prime}}=C^{\prime}+K_{1} X$ and upper bounds $\mathrm{t}_{R}=C_{l}+K X$ and $\mathrm{t}_{R^{\prime}}$ $=C_{I}{ }^{\prime}+K_{l} X^{\prime}$. The time interval for the result is defined as $\mathrm{t}_{L}=C^{\prime \prime}+K^{\prime} X$ the common lower bound where $C^{\prime}=\max$ $\left(C, C^{\prime}\right)$, and $K^{\prime}=\min \left(K, K_{l}\right)$, and $\mathrm{t}_{U^{\prime}}=C_{3}+K^{\prime} X$ the common upper bound where $C_{3}=\max \left(C_{l} C_{1}{ }^{\prime}\right)$, and $K^{\prime}=$ $\min \left(K, K_{l}\right)$.

Union: Union compatibility means that two extended relations $R, S$ are union compatible if and only if they 
have the same arity or degree and their corresponding attributes are based on the same domain.

\begin{tabular}{|c|c|c|c|}
\hline $\mathrm{R}$ & Person & Concept & $\mathrm{VT}(\mathrm{R})$ \\
\hline$X_{1}$ & Ann & Brazil & $\begin{array}{l}{\left[\mathrm{K} \times \mathrm{X}+\mathrm{C}_{1}, \mathrm{~K} \times \mathrm{X}+\right.} \\
\left.\mathrm{C}_{1^{\prime}}\right], \mathrm{X}=0, \mathrm{~K}=0, \mathrm{C}_{1^{-}} \\
\mathrm{C}_{1}^{\prime}=\mathrm{D}, \mathrm{D}=90\end{array}$ \\
\hline$X_{2}$ & Ann & $\begin{array}{l}\text { Southern } \\
\text { Hemisphere }\end{array}$ & $\begin{array}{l}{\left[\mathrm{K} \times \mathrm{X}+\mathrm{C}_{2}, \mathrm{~K} \times \mathrm{X}+\right.} \\
\left.\mathrm{C}_{2}^{\prime}\right], \mathrm{X}=0, \mathrm{~K}=0, \mathrm{C}_{2^{-}} \\
\mathrm{C}_{2}^{\prime}=\mathrm{D}, \mathrm{D}=90\end{array}$ \\
\hline$X_{3}$ & Ann & $\begin{array}{l}\text { Northern } \\
\text { Hemisphere }\end{array}$ & $\begin{array}{l}{\left[\mathrm{K} \times \mathrm{X}+\mathrm{C}_{3}, \mathrm{~K} \times \mathrm{X}+\right.} \\
\left.C_{3}{ }^{\prime}\right], 0 \leq \mathrm{X} \leq 1, \mathrm{~K}=90, C_{3^{-}} \\
\mathrm{C}_{3}{ }^{\prime}=\mathrm{D}, \mathrm{D}=90\end{array}$ \\
\hline $\mathrm{X}_{4}$ & Ann & Brazil & $\begin{array}{l}{\left[\mathrm{K} \times \mathrm{X}+\mathrm{C}_{4}, \mathrm{~K} \times \mathrm{X}+\right.} \\
\left.\mathrm{C}_{4}{ }^{\prime}\right], 0 \leq \mathrm{X} \leq \mathrm{N}, 90 \leq \mathrm{K} \leq \mathrm{M}, \\
\mathrm{C}_{4}-\mathrm{C}_{4}{ }^{\prime}=\mathrm{D}, \mathrm{D}=90\end{array}$ \\
\hline $\mathrm{X}_{5}$ & Liz & Brazil & $\begin{array}{l}{\left[\mathrm{K} \times \mathrm{X}+\mathrm{C}_{5}, \mathrm{~K} \times \mathrm{X}+\right.} \\
\left.\mathrm{C}_{5}^{\prime}\right], \mathrm{X}=0, \mathrm{~K}=0, \mathrm{C}_{5^{-}} \\
\mathrm{C}_{5}{ }^{\prime}=\mathrm{D}, \mathrm{D}=90\end{array}$ \\
\hline$\overline{X_{6}}$ & $\mathrm{Liz}$ & $\begin{array}{l}\text { Northern } \\
\text { Hemisphere }\end{array}$ & $\begin{array}{l}{\left[\mathrm{K} \times \mathrm{X}+\mathrm{C}_{6}, \mathrm{~K} \times \mathrm{X}+\right.} \\
\left.\mathrm{C}_{6}{ }^{\prime}\right], 0 \leq \mathrm{X} \leq 1, \mathrm{~K}=90, \mathrm{C}_{6}- \\
\mathrm{C}_{6}{ }^{\prime}=\mathrm{D}, \mathrm{D}=90\end{array}$ \\
\hline$\overline{X_{7}}$ & $\mathrm{Liz}$ & Brazil & $\begin{array}{l}{\left[\mathrm{K} \times \mathrm{X}+\mathrm{C}_{7}, \mathrm{~K} \times \mathrm{X}+\right.} \\
\left.\mathrm{C}_{7}^{\prime}\right], 0 \leq \mathrm{X} \leq \mathrm{N}, 90 \leq \mathrm{K} \leq \mathrm{M}, \\
\mathrm{C}_{7}-\mathrm{C}_{7}=\mathrm{D}, \mathrm{D}=90\end{array}$ \\
\hline
\end{tabular}

"Table 2: The Proposed Model Representation"

For the set operators, including union, uncertainty can be introduced when relations with different levels of refinement for the same information are combined. Without extra knowledge it is reasonable to choose the information with the finest granularity as the one to be classified with full certainty $(B e l, P l s)=[1,1]$. Information not in the finest granularity is classified with no full certainty $(\mathrm{Bel}, \mathrm{Pls})=(0,1]$. Both types of information are part of the result tuples, accompanied by different beliefs. Union is formally defined as follows

$R \cup S \equiv\{\mathrm{tl}(\exists \mathrm{r})(\exists \mathrm{s})(\mathrm{r} \in R \wedge \mathrm{s} \in S \wedge \mathrm{t} . K=\mathrm{r} . K=\mathrm{s} . K) \wedge$ $\left(\mathrm{t}(\mathrm{Bel}, \mathrm{Pls})=\mathrm{F}_{\mathrm{s}}(\mathrm{r} .(\mathrm{Bel}, \mathrm{Pls}), \mathrm{s}(\mathrm{Bel}, \mathrm{Pls}))\right\} . K$ is the arity of the relation, $F_{\mathrm{s}}$ denotes the selection support function. Tuples with different valid times are not merged, independently of the fact that they are expressing the same snapshot tuple.At this point we will try to suggest ways of incorporating in the initial set of answers, new results coming from new contributions assuming that the number of information providers is not static.

\section{Conclusions - Open Issues}

It is considered that in a virtual integrated environment the community of member databases is not stable. Frequent changes in this community take place with new information providers added or existing ones modified and deleted. The target is to design algorithms for projective and selective transformation defined as:

Projective transformation: Discover the columns of the new contribution with respect to the validity and the semantics of the initial view.

Selective transformation: Discover new tuples of the new contribution using the results of the projective transformation using a membership threshold, without destroying the validity and the semantics of our initial view.

In both stages of discovery the likelihood of a successful discovery must be estimated and eventually all new tuples must be tagged with a level of confidence. The main focus currently is on the inclusion of information coming from new sources in a non static database environment.

\section{References}

1. Widom J. Integrating Heterogeneous Databases: Lazy or Eager?. ACM Computing Surveys, 28A(4), December 1996. Invited short position paper for ACM Workshop on Strategic Directions in Computing Research

2. Motro A, Anokhin P, Berlin J, Intelligent Methods in Virtual Databases, Proceedings of the Fourth International Conference on Flexible Query Answering Systems, FQAS 2000, Physica-Verlag Heidelberg, October, 2000, Warsaw Poland

3. Hull R, Managing Semantic Heterogeneity in Databases: A Theoretical Perspective. PODS 1997: 51-61, Proceedings of the Sixteenth ACM SIGACT-SIGMOD-SIGART Symposium on Principles of Database Systems, May 12-14, 1997, Tucson, Arizona

4. ' Lim E, Sristava, J, Prabhakar S, Richardson J, Entity Identification Problem in Database Integration, Proceedings of the $9^{\text {th }}$ IEEE, Data Engineering Conference, IEEE Computer Society Press April 1993, Vienna, Austria

5. Chountas, P, Petrounias, I., Representation of Definite, Indefinite and Infinite Temporal Information, Proceedings of the 4th International Database Engineering \& Applications Symposium (IDEAS'2000), IEEE Computer Society Press, September 2000, Japan.

6. Koubarakis $\mathbf{M}$, Database Models for Infinite and Indefinite Temporal .Information, Information Systems, Vol. 19, No. 2, pages 141-173, 1994

7. Chountas, P, Petrounias, I., Modelling and Representation of Uncertain Temporal Information, Requirements Engineering Journal, 5(3), pp 144-156, Springer Verlag, 2000.

8. Lakshmanan L.V.S, Leone R, Subrahmanian V. S, ProbView: A Flexible Probabilistic Database System, ACM Transactions on Database Systems, Volume 22, No. 3 (Sep. 1997)

9. Baldwin J. F, Evidential Support Logic Programming" Fuzzy sets and Systems, 24, pp 1-26, 1987 